\title{
Sweet Syndrome in an Adolescent Patient With Differentiation Syndrome Secondary to Promyelocytic Leukemia Treatment With All-Trans Retinoic Acid
}

\author{
Jorge Luis Ramirez Melo a, d, Rosa Margarita Cruz Osorio ${ }^{\text {a }}$, Jessica Santoyo Cueva ${ }^{a}$, \\ Fernando Sanchez Zubieta ${ }^{a}$, Pablo Alejandro Chavez ${ }^{\mathrm{a}}$, Luis Tonatiuh Fernandez Mendoza ${ }^{\mathrm{b}}$, \\ Felipe de Jesus Bustos Rodriguez ${ }^{\mathrm{b}}$, Christian David Burbano Figueroa ${ }^{\mathrm{c}}$, \\ Johana Alexandra Burbano Figueroa ${ }^{c}$
}

\begin{abstract}
Sweet syndrome (SS) is an acute febrile neutrophilic dermatosis that is histologically characterized by an infiltration of the dermis by neutrophils. A 12-year-old adolescent female patient recently diagnosed with acute promyelocytic leukemia presented with fever and was hospitalized for antibiotic management after 22 days of being treated with a treatment protocol based on daunorubicin, all-trans retinoic acid (ATRA), and prophylaxis with dexamethasone, the patient developed erythematous skin lesions located mostly on the extremities. Lesions evolved into painful subcutaneous nodules, and one lesion evolved into a $2.5-\mathrm{cm}$ blister with a purple and necrotic base. A skin biopsy was performed and showed neutrophilic dermatosis which confirmed the diagnosis of SS. The patient's clinical features complied with criteria for differentiation syndrome complicated by shock. Two days after ATRA was suspended, the patient presented resolution of the fever and skin lesions. SS is a rare neutrophilic dermatosis secondary to an innate immune disorder classified into four categories: classical (idiopathic), para-inflammatory, paraneoplastic or pregnancy-related. SS has been described in patients with acute myeloid leukemia in adults secondary to the use of drugs such as ATRA or as a part of a paraneoplastic syndrome. SS can occur exceptionally in children with myeloid leukemia secondary to the use of drugs such as ATRA.
\end{abstract}

Keywords: Sweet syndrome; Dermatosis; Promyelocytic leukemia; Children

Manuscript submitted August 19, 2021, accepted September 18, 2021

Published online December 2, 2021

aPediatric Department, Pediatric Oncology-Hematology Division, Hospital Civil de Guadalajara "Dr. Juan I. Menchaca", Guadalajara, Jalisco, Mexico bPathological Anatomy Department, Hospital Civil de Guadalajara "Dr. Juan I. Menchaca", Guadalajara, Jalisco, Mexico

${ }^{c}$ Hospital Universitario San Jose de Popayan, Cauca, Colombia

${ }^{\mathrm{d} C}$ Corresponding Author: Jorge Luis Ramirez Melo, Pediatric Department, Pediatric Oncology-Hematology Division, Hospital Civil de Guadalajara "Dr Juan I. Menchaca", Guadalajara, Jalisco, Mexico.

Email: georgerluis117@hotmail.com

doi: https://doi.org/10.14740/jmc3758

\section{Introduction}

Sweet syndrome (SS) is an acute febrile neutrophilic dermatosis that manifests as a febrile syndrome associated with neutrophilia and skin lesions that are histologically reported as a dermis infiltration of neutrophils. In $72 \%$ of the cases, it is of idiopathic origin. However, drug administration and paraneoplastic syndromes have been described as triggering factors $[1$, 2]. The objective of this case report is to describe a secondary SS at the start of induction chemotherapy in a patient with acute promyelocytic leukemia (APL).

\section{Case Report}

\section{Investigations}

A 12-year-old female patient with no significant medical history presented to the emergency department with a chief complaint of 1-month history of global headache of mild intensity that improved with the use of oral analgesics and weight loss of $2 \mathrm{~kg}$. Two weeks later, she presented with fever and recurrent epistaxis despite the use of cauterization of the nasal vessels with silver nitrate. Hematic biometry was performed that reported platelets of $48,200 / \mu \mathrm{L}$, hemoglobin of $13.3 \mathrm{~g} / \mathrm{dL}$, leukocytes of 4,600 cells $/ \mu \mathrm{L}$, and neutrophils of 1,720 cells $/ \mu \mathrm{L}$, and a peripheral blood smear showed abnormal promyelocytes. The patient was then referred to a hematology service. The physical examination did not report any evidence of bleeding and laboratory tests were performed ruling out coagulopathy. Bone marrow aspirate and flow cytometry immunophenotype were performed, which were compatible with hypogranular variant APL. Fluorescence in situ hybridization (FISH) reported negative to a $\mathrm{t}(15 ; 17)$ translocation and no polymerase chain reaction was performed. The patient received the first cycle of induction chemotherapy using the Treatment Protocol of Hematological Malignancies from the Spanish Society of Hematology (PETHEMA 12), based on daunorubicin, all-trans retinoic acid (ATRA) and prophylaxis with dexamethasone. After 22 days, the patient was hospitalized for fever, asthenia 

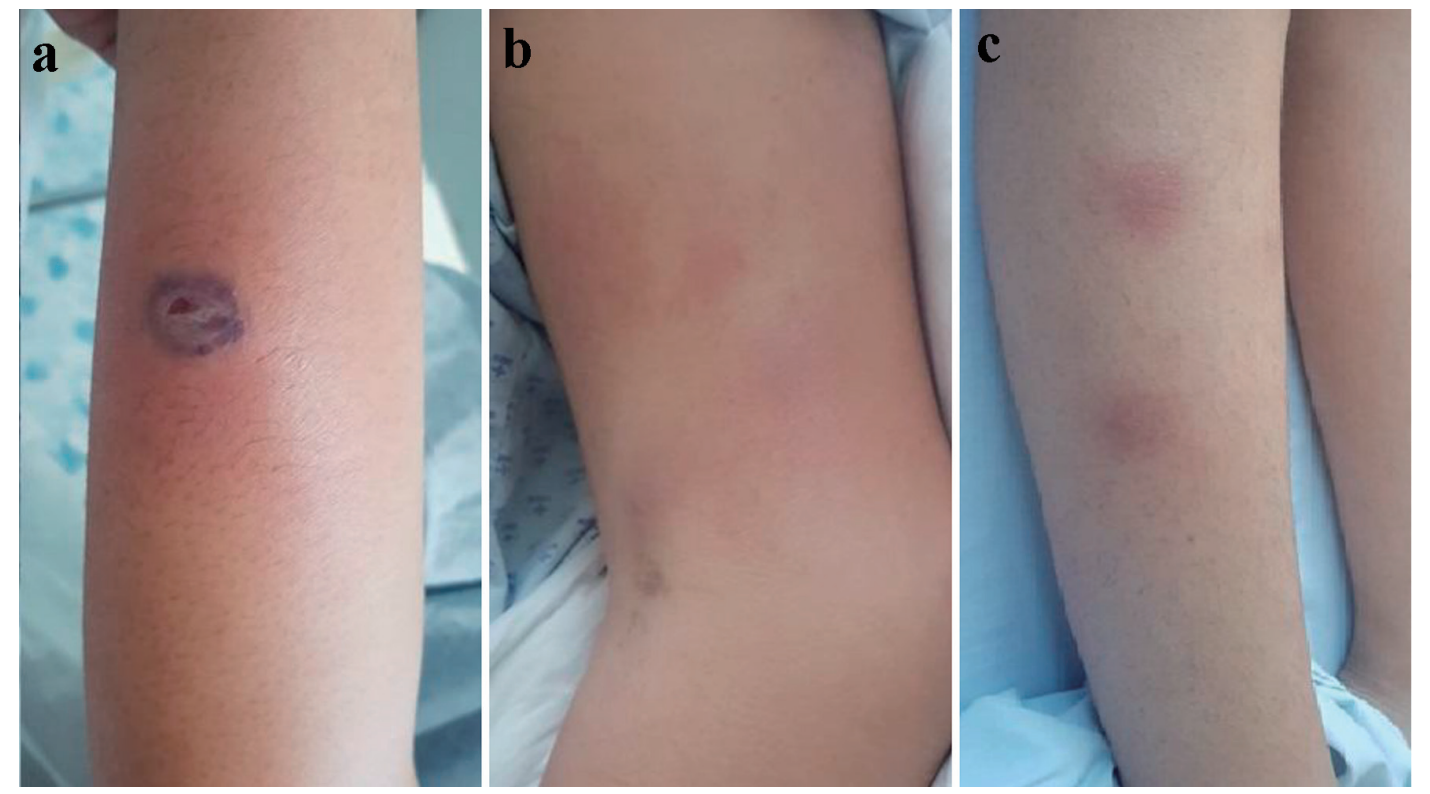

Figure 1. (a) One centimeter purplish-based blister in the middle-third of left forearm. (b) Erythematous lesions in the middle and distal-third of left forearm. (c) Erythematous subcutaneous nodules in the middle-third of right leg.

and adynamia and was initially treated with a 4-day antibiotic regimen using cefepime and amikacin. Antibiotic management was changed to vancomycin, meropenem and colistin due to her persistent fever pattern. A negative culture was reported. Upon clinical examination, the patient presented with non-pruritic erythematous skin lesions predominantly found in lower limbs, which had local heat (Fig. 1a, b). Lesions progressed into subcutaneous nodules that were painful to the touch. One of lesions evolved into a $2.5-\mathrm{cm}$ blister with a violaceous and necrotic base (Fig. 1c). Simultaneously, the patient developed a global headache of moderate intensity which improved with the administration of analgesics. No neurological deficit was shown. Dyspnea and bibasal rales were also present. A chest radiograph was performed, which showed bilateral interstitial infiltrates. Two days after the appearance of the skin lesions, the patient reported intense pain in the left upper limb associated with progressive edema along the peripheral catheter. A Doppler ultrasound was performed which revealed thrombosis of the axillary and basilic vein in the left arm. Anticoagulation therapy with enoxaparin was started which resolved the thrombosis. Antibiotic management was followed after the presentation of cellulitis.

\section{Diagnosis}

Given the persistent pattern of the febrile picture with a negative culture report and the skin lesions, SS was suspected. Treatment with ATRA was suspended and a skin biopsy was performed, confirming neutrophilic dermatosis (Fig. 2a-c).

\section{Treatment}

The patient met the diagnostic criteria for ATRA differentia- tion syndrome (DS); therefore, treatment was suspended. Two days after the drug was suspended, the patient presented resolution of the fever and skin lesions resolved a week later leaving hyperpigmented skin patches.

\section{Follow-up and outcomes}

Treatment with ATRA was resumed 7 days later without complications.

\section{Discussion}

SS is an acute febrile neutrophilic dermatosis that presents as a febrile syndrome associated with neutrophilia and skin lesions that are histologically characterized by dermis infiltration of neutrophils [3, 4]. Dr. Robert Douglas Sweet described this pathology for the first time in 1964 in eight women who presented with a clinical picture with four common characteristics: fever, leukocytosis, painful skin lesions and a skin infiltrate by mature neutrophils [5]. Over the course of 22 years, the dermatology program at Boston Children's Hospital treated seven patients with SS and found a higher frequency of appearance in females [4]. There is no increase in prevalence according to race [6]. Neutrophilic dermatosis consists of a large group of diseases in which skin lesions arise as a result of an immunologically mediated hypersensitivity phenomenon that cause loss of neutrophil infiltration regulation [7,8].

Interleukin (IL)-1 $\beta$ plays an important role in infectious and inflammatory processes and it is believed that it is also involved in patients with SS since high levels have been reported.

In addition, high levels of interferon- $\gamma$ and IL-2 have also 

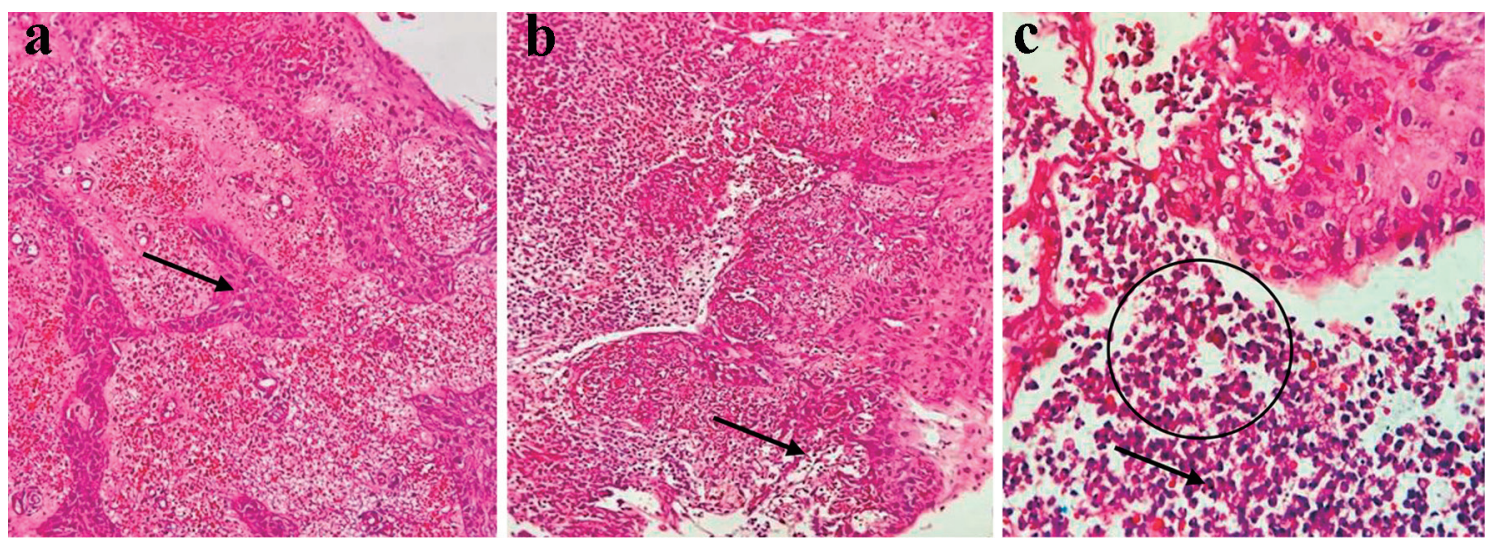

Figure 2. Histopathological study. (a) Dense inflammatory infiltrate composed of neutrophils and abundant extravasation of erythrocytes in the dermis. Only mild spongiosis (arrow) is observed in the epidermis (hematoxylin and eosin, $\times 10$ ). (b) Dense neutrophilic inflammatory infiltrate, abundant cellular debris and formation of papillary micro abscesses (arrow) (hematoxylin and eosin, $\times 10$ ). (c) Neutrophilic inflammatory infiltrate (circle), cells with multilobulated nuclei and less eosinophilic cytoplasm (arrow), abundant cellular debris, and scattered extravasated erythrocytes (hematoxylin and eosin, $\times 10$ ).

Table 1. Factors Associated With Sweet Syndrome Modified From Reference [4]

\begin{tabular}{|ll}
\hline Paraneoplastic & Acute lymphoid leukemia \\
& Acute myeloid leukemia \\
& Juvenile myelomonocytic leukemia \\
& Myelodysplastic syndromes \\
& Osteosarcoma \\
& Fanconi anemia \\
& Aplastic anemia \\
\hline Inflammatory diseases & Systemic lupus erythematosus \\
& Auto immune hepatitis \\
\hline Infections & Crohn's disease \\
& Human immunodeficiency virus \\
& Rotavirus \\
& Otitis media \\
& Tonsilitis \\
& Acute respiratory infections \\
Drug treatment & Colony stimulating factors \\
& Trimethoprim sulfamethoxazol \\
& Retinoids \\
& Azathioprine \\
& Contraceptives \\
\hline Pregnancy & Minocycline \\
\hline & Carbamazepine \\
\hline Vaccines & Tyrosine kinase inhibitors \\
\hline & BCG \\
\hline & Measles \\
\hline & Influenza \\
\hline & Pneumococcus \\
\hline
\end{tabular}

BCG: bacille Calmette-Guerin. been found [9].

In $72 \%$ of the cases, SS is of idiopathic origin [1]; however, triggering factors have been described and are summarized in Table $1[1,2]$. In secondary SS, the most common causes are infectious and autoimmune processes and vaccine administrations. SS is secondary to cancer in $11 \%$ of the cases [10]. In hematological neoplasms, it has been described as a manifestation when leukemia is first diagnosed or when ATRA treatment is started in patients with APL $[1,2]$.

Up to $80 \%$ of SS cases secondary to malignant processes are related to acute myeloid leukemia and myelodysplastic syndromes [10].

The most frequent drugs associated with SS are colonystimulating factors. Twenty-six cases have reported in literature, of which 13 cases have been associated with ATRA. Minocycline has been reported to be less frequent [5]. Recently, SS has also been described as an adverse effect of tyrosine kinase inhibitors [11].

In order to diagnose SS, the criteria proposed in 1986 by $\mathrm{Su}$ and Liu are taken into account: two major and two minor criteria must be met [12]. These are described in detail in Table 2 [12].

Clinically, SS is characterized by the appearance of nodular, papular, plaque lesions, or violaceous bullae with an asymmetric distribution associated with general symptoms such as joint pain, myalgia and in severe cases airway obstruction [13, 14]. Systemic manifestations of SS are not frequent. However, pericarditis, valvular damage, myocardial infarction and dilation of the great vessels have been described at the cardiac level [15]. Systemic involvement has been exceptionally reported with neutrophilic infiltrate in bones, central nervous system, liver, gastrointestinal tract, spleen, lymph nodes, cardiovascular system and eyes [16]. SS has been described by some authors as a secondary manifestation of a DS and not as a different entity [17]. In this reported clinical case, the patient met criteria for DS and SS. Infiltration of the subcutaneous cellular tissue is frequent and is characterized by the presence of nodules located on the anterior part of the legs [6]. The devel- 
Table 2. Diagnostic Criteria Proposed by Su and Liu in 1986 and Modified by Von Den Driesch in 1992 [12]

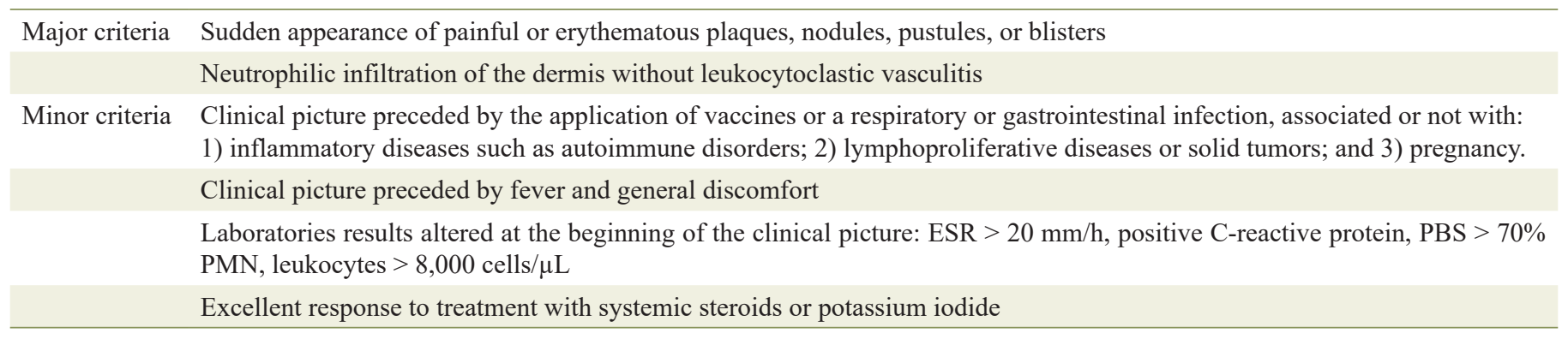

ESR: erythrocyte sedimentation rate; PBS: peripheral blood smear; PMN: polymorphonuclear.

opment of lobular panniculitis has been described as part of SS in patients with APL receiving ATRA [18]. In cases associated with neoplastic pathologies, recurrence of the disease may occur in up to $45 \%$ of patients while not receiving treatment [3].

The use of ATRA in treating APL was historically one of the most influential factors that improved prognosis and survival rate of patients. Upon its administration, there is a massive release of cytokines such as IL-1, IL-8 and tumor necrosis factor (TNF)- $\alpha$, which may be associated with complications such as DS, pseudotumor cerebri, SS, scrotal ulcers, and Fournier's gangrene [19]. SS in patients receiving treatment with ATRA for APL is a clinical marker of effectiveness of the drug to successfully induce promyelocyte maturation [18]. Generally, patients with SS present neutrophilia; however, if associated with a malignant hematological disease, cytopenias may occur [20]. Histologically, infiltration of the dermis by neutrophils and subdermal edema without leukocytoclastic vasculitis is observed [13]. The main finding is an intense neutrophilic infiltrate predominantly in the reticular dermis, and the density can simulate the histology of an abscess with abundant cellulite detritus areas. Leukocytoclasia is less severe than that seen in vasculitis and there is edema of the endothelium with extravasation of erythrocytes. The epidermis is usually normal, but occasionally there may be slight spongiosis, formation of vesicles and pustules [21]. Differential diagnoses by pathology are pyoderma gangrenosum in which ulcers may be observed, deeper inflammation and less prominent karyorrhexis than in SS, infections, leukocytoclastic vasculitis in which there is necrosis of the vascular walls, fibrin deposits and perivascular neutrophilic infiltrate $[22,23]$. The first line of treatment for SS is systemic steroids with doses equivalency of prednisone of $1-2 \mathrm{mg} / \mathrm{kg} /$ day. In SS secondary to drugs, the triggering drug should be suspended [2]. The response to steroids and the interruption of the triggering drug strongly supports the diagnosis of SS. If SS is secondary to ATRA (in our case, we considered it to be the triggering factor), this drug can be reintroduced into the treatment protocol without complications [14].

\section{Learning points}

SS can occur exceptionally in children with APL secondary to the start of induction chemotherapy that includes ATRA.

The presence of sudden onset skin lesions during induction treatment of patients with myeloid leukemia should prompt clinicians to suspect this complication and it is mandatory to perform a skin biopsy. If a skin biopsy reports neutrophilic infiltration of the dermis without leukocytoclastic vasculitis, the diagnosis of SS can be confirmed.s

\section{Acknowledgments}

None to declare.

\section{Financial Disclosure}

This research has not received any specific grant from public agencies, commercial or non-profit sectors.

\section{Conflict of Interest}

The authors declare that they have no conflict of interest.

\section{Informed Consent}

A summary of the clinical history was done prior to the signing of the informed consent.

\section{Author Contributions}

Jorge Luis Ramirez Melo, Rosa Margarita Cruz Osorio and Jessica Santoyo Cueva provided the conception and design of the study, acquisition of data, analysis and interpretation of data, drafting the article, revised it critically for important intellectual content, and final approval of the version to be submitted. Luis Tonatiuh Fernandez Mendoza, Johana Burbano Figueroa and Felipe de Jesus Bustos Rodriguez were responsible the acquisition of data, drafting of manuscript. Christian David Burbano Figueroa and Pablo Alejandro Chavez were responsible for the article critically for important intellectual content. Fernando Sanchez Zubieta provided the revised the article critically for important intellectual content and gave final approval of the version to be submitted. 


\section{Data Availability}

The authors declare that data supporting the findings of this study are available within the article and the data supporting the findings of this study are available from the corresponding author upon request by email too.

\section{References}

1. Astudillo L, Loche F, Reynish W, Rigal-Huguet F, Lamant L, Pris J. Sweet's syndrome associated with retinoic acid syndrome in a patient with promyelocytic leukemia. Ann Hematol. 2002;81(2):111-114.

2. Hospach T, von den Driesch P, Dannecker GE. Acute febrile neutrophilic dermatosis (Sweet's syndrome) in childhood and adolescence: two new patients and review of the literature on associated diseases. Eur J Pediatr. 2009;168(1):1-9.

3. Stevens GJ, Yutronic HJ, Pizarro OJ, Velozo PL. Sweet syndrome in pediatrics. A case report. Rev Chil Pediatr. 2018;89(4):511-515.

4. Uihlein LC, Brandling-Bennett HA, Lio PA, Liang MG. Sweet syndrome in children. Pediatr Dermatol. 2012;29(1):38-44.

5. Thompson DF, Montarella KE. Drug-induced Sweet's syndrome. Ann Pharmacother. 2007;41(5):802-811.

6. Ginarte M, Toribio J. Sweet syndrome. Dermatol Online J. 2011;9(4):96-110.

7. Satoh TK, Mellett M, Contassot E, French LE. Are neutrophilic dermatoses autoinflammatory disorders? Br J Dermatol. 2018;178(3):603-613.

8. Fernandez-Torres RM, Castro S, Moreno A, Alvarez R, Fonseca E. Subcutaneous histiocytoid sweet syndrome associated with crohn disease in an adolescent. Case Rep Dermatol Med. 2014;2014:954254.

9. Webb K, Hlela C, Jordaan HF, Suliman S, Scriba T, Lipsker D, Scott C. A review and proposed approach to the neutrophilic dermatoses of childhood. Pediatr Dermatol. 2015;32(4):437-446.

10. Merlant M, Lepelletier C, Battistella M, Vignon-Pennamen MD, Duriez P, Moguelet P, Brunet-Possenti F, et al. Acute myeloid leukemia and myelodysplastic syndromeassociated Sweet syndrome: A comparative multicenter retrospective study of 39 patients. J Am Acad Dermatol. 2021;84(3):838-840.
11. Yang JJ, Maloney NJ, Nguyen KA, Worswick S, Smogorzewski J, Bach DQ. Sweet syndrome as an adverse reaction to tyrosine kinase inhibitors: A review. Dermatol Ther. 2021;34(1):e14461.

12. von den Driesch P. Sweet's syndrome (acute febrile neutrophilic dermatosis). J Am Acad Dermatol. 1994;31(4):535556; quiz 557-560.

13. Makis A, Stavrou S, Chaliasos N, et al. Acute febrile neutrophilic dermatosis (Sweet's syndrome) in a child, associated with a rotavirus infection: a case report. J Med Case Rep. 2010;4(1):2-7.

14. Arnold KA, Gao J, Stein SL. A review of cutaneous hypersensitivity reactions in infants: From common to concerning. Pediatr Dermatol. 2019;36(3):274-282.

15. Swetha P, Prasad P, Kaviarasan P. Sweet's syndrome in the pediatric population: Two case reports. Indian J Paediatr Dermatology. 2015;16(3):179.

16. Parsapour K, Reep MD, Gohar K, Shah V, Church A, Shwayder TA. Familial Sweet's syndrome in 2 brothers, both seen in the first 2 weeks of life. J Am Acad Dermatol. $2003 ; 49(1): 132-138$.

17. Stahl M, Tallman MS. Differentiation syndrome in acute promyelocytic leukaemia. Br J Haematol. 2019; 187(2):157-162.

18. Jagdeo J, Campbell R, Long T, Muglia J, Telang G, Robinson-Bostom L. Sweet's syndrome-like neutrophilic lobular panniculitis associated with all-trans-retinoic acid chemotherapy in a patient with acute promyelocytic leukemia. J Am Acad Dermatol. 2007;56(4):690-693.

19. Jeddi R, Kacem K, Ben Neji H, Mnif S, Gouider E, Aissaoui L, Ben Amor R, et al. Predictive factors of alltrans-retinoic acid related complications during induction therapy for acute promyelocytic leukemia. Hematology. 2008;13(3):142-146.

20. Santos TB, Sales BC, Sigres M, Rosman F, Cerqueira AM. Sweet Syndrome in childhood. An Bras Dermatol. 2015;90(4):567-569.

21. Hamodat M. Skin nontumor. Dermal non-granulomatous granulocyte-rich reaction patterns Sweet syndrome. www. pathologyoutlines.com/topic/skinnontumorsweetssyndrome.html. Published 2020. Accessed March 16, 2021.

22. Hall B, Chisholm C, Vandergriff T, Jessup C. Diagnostic pathology: Nonneoplastic Dermatopathology, 2nd Ed. 2016; Vol 77.

23. Cardoso J. Practical dermatopathology, 2nd Ed. 2012; Vol 7. 